\title{
A Síndrome Painful Legs and Moving Toes Associada a Alterações nos Núcleos da Base
}

\author{
Thaís S. C. Minett ${ }^{1}$ \\ Orlando G. P. Barsottini ${ }^{2}$ \\ Vanderci Borges ${ }^{3}$ \\ Henrique Ballalai Ferraz ${ }^{4}$
}

\begin{abstract}
RESUMO
Apresentamos o caso de uma paciente com síndrome painful legs and moving toes. A paciente realizou eletroneuromiografia (ENMG), tomografia de crânio (TC) e ressonância magnética de encéfalo (RM). A ENMG não mostrou anormalidades, porém o padrão dos movimentos anormais foi sugestivo de quadro periférico. Os exames de imagens mostraram atrofia dos núcleos da base à esquerda e lesões hipodensas na substância branca sugestiva de infartos lacunares. Sendo possível a causa periférica ou central como responsável pelo quadro, sugerimos que a combinação dos dois fatores também deva ser considerada como mais um fator etiológico. A paciente apresentou uma resposta satisfatória com o uso de carbamazepina, diminuindo o quadro álgico e também os movimentos involuntários.
\end{abstract}

Unitermos: Pernas dolorosas, movimentos involuntários, núcleos da base.

\section{Introdução}

A síndrome painful legs and moving toes (PLMT) foi descrita pela primeira vez por Spillane et al. ${ }^{1}$. Essa síndrome consiste em uma associação de movimentos involuntários dos artelhos e dos pés com dor no membro inferior afetado. O espectro doloroso é amplo, variando de um desconforto permanente até uma dor intensa do tipo causalgia, mas sem os componentes simpaticorreflexos. Também são observados casos que não apresentam dor ${ }^{2}$. Esta condição, sem dor, é conhecida como síndrome painless legs and moving toes, descrita por Walters et al. ${ }^{3}$. Os movimentos involuntários são caracterizados pela combinação de flexão e extensão e de adução e abdução dos artelhos, do tipo atetose, e podem ser suprimidos voluntariamente $^{1}$. Em geral, a dor precede o quadro de movimentos involuntários, mas o inverso também pode ocorrer. Dressler et al. ${ }^{4}$ descreveram 23 pacientes com essa síndrome, e 18 tiveram como primeira mani- festação o quadro álgico. Entre os cinco outros pacientes, apenas em dois os movimentos precederam a dor e nos outros três a dor não ocorreu. Os sintomas podem começar de um lado e, posteriormente, tornarem-se bilaterais.

Quanto a etiopatogenia, o trauma da medula espinhal ou da cauda eqüina, a lesão de raízes lombares, o trauma ósseo ou de partes moles dos pés e a neuropatia periférica têm um papel importante em alguns casos $^{1,4,5,6,7,8}$. Processos infecciosos também foram associados ao quadro ${ }^{8}$ e o aparecimento dessa síndrome induzida por drogas ocorreu com o uso concomitante da vincristina com o metronidazol ${ }^{9}$, e da citosina arabinoside (Ara C) ${ }^{10}$. O objetivo deste trabalho é apresentar o caso de um paciente portador de PLMT com evidência de envolvimento encefálico e periférico simultaneamente, uma combinação ainda não descrita, e discutir a possível correlação etiopatogênica desses achados.

Pós-graduanda de Neurologia da Escola Paulista de Medicina - Unifesp.

Mestre em Neurologia da Escola Paulista de Medicina - Unifesp.

Doutora em Neurologia da Escola Paulista de Medicina - Unifesp.

Doutor em Neurologia e Chefe do Setor de Distúrbios do Movimento da Disciplina de Neurologia da Escola Paulista de Medicina Unifesp. 


\section{Descrição de caso}

Paciente do sexo feminino, de 55 anos, obesa e hipertensa, procurou Hospital São Paulo por ter apresentado três episódios de ataques isquêmicos transitórios manifestados, clinicamente, por paresia e parestesia, ora do membro superior direito ora do lado oposto, com regressão espontânea e completa em cinco minutos. Notou-se o aparecimento de movimentos involuntários nos artelhos à direita e dor no membro inferior do mesmo lado, simultaneamente com o início de sintomatologia vascular há um mês e meio. A dor era de moderada intensidade, localizada na panturrilha direita, tinha um caráter de queimação, intermitente, sem relação com os movimentos involuntários e sem fenômenos simpáticos acompanhando. Ao exame, notava-se uma paciente obesa com pressão arterial de $160 \mathrm{mmHg}$ x $80 \mathrm{mmHg}$. Apresentava movimentos de flexão e extensão de todos os artelhos e hálux acompanhados ou não de flexão e extensão do pé direito, arrítmicos, intermitentes, de pequena amplitude, não suprimidos voluntariamente, que pioravam com a distração da paciente. A força muscular era normal, os reflexos profundos hipoativos, globalmente, sem alterações semiológicas em pares cranianos, provas de sensibilidade superficial e profunda normais.

Após um mês, o membro inferior esquerdo também foi acometido, porém com menor intensidade. A investigação com exames laboratoriais de rotina revelaram que a paciente era diabética com glicemia de jejum de $120 \mathrm{mg} / \mathrm{dl}$ e com teste de tolerância oral à glicose confirmando o achado.

Foi submetida à eletroneuromiografia (ENMG), que não mostrou sinais típicos de uma neuropatia periférica, mas que revelou descargas espontâneas, esporádicas, de freqüência irregular, baixa amplitude, vistas sincronicamente em músculos antagonistas que sugeriam um envolvimento periférico (Figura 1). O estudo Doppler fluxométrico das carótidas evidenciou velocidades sistólicas e diastólicas normais, bilateralmente. $\mathrm{Na}$ tomografia computadorizada de crânio (TC) observamos uma assimetria de núcleos caudados e, no lado esquerdo, sinais de atrofia. Na ressonância nuclear magnética (RNM) esses achados foram mais evidentes, em que pudemos observar também uma discreta atrofia dos núcleos da base à esquerda e mínimas lesões na substância branca à direita, sugerindo pequenas áreas de isquemia e gliose (Figura 2).

Foi prescrita carbamazepina na dose de $200 \mathrm{mg} / \mathrm{dia}$. Após um mês de terapia a paciente referiu melhora do quadro álgico e uma diminuição dos movimentos, porém sem remissão completa do quadro.

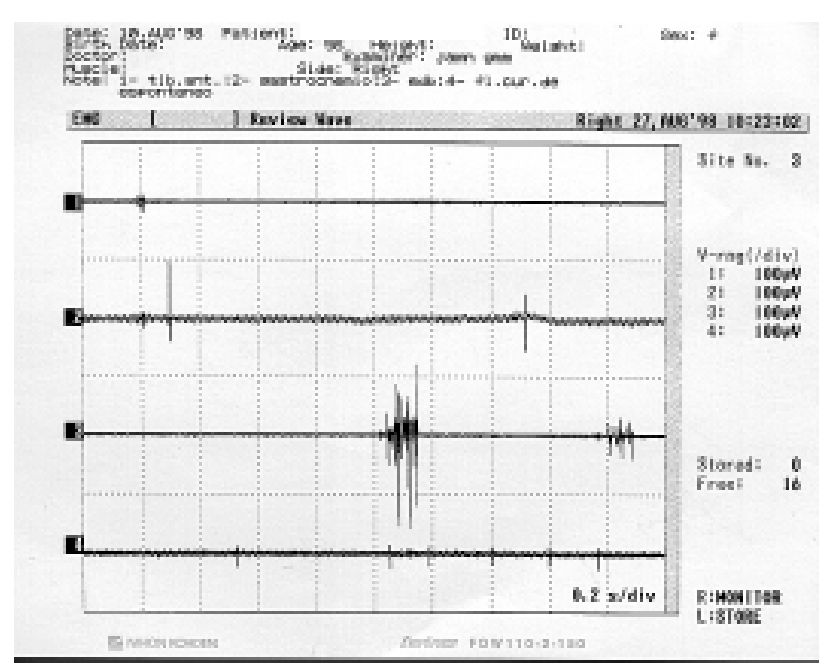

Figura 1

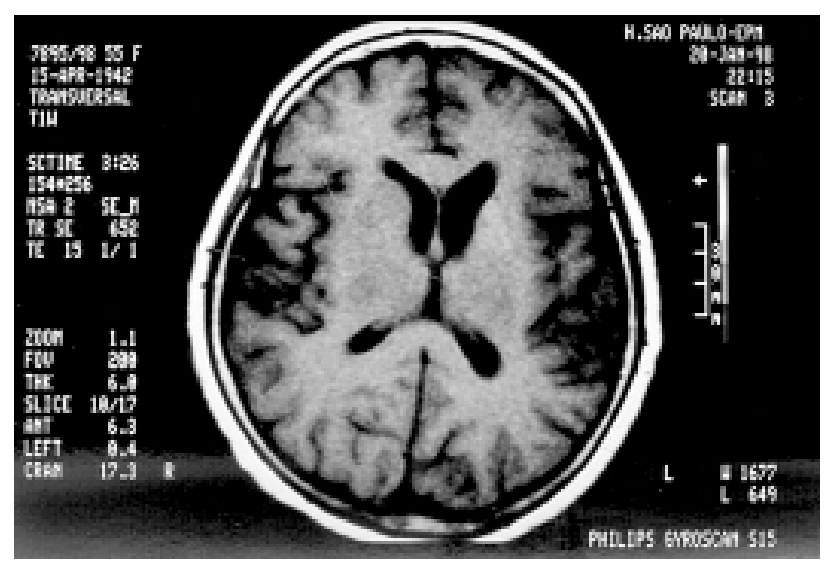

Figura 2 Imagem de ressonância magnética em que observamos, como principal achado, a assimetria dos núcleos caudados e, no lado esquerdo, sinais de atrofia.

\section{Discussão}

Existem diversas descrições sugerindo o sítio de lesão nos pacientes com PLMT: podemos encontrar

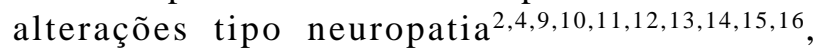
plexopatia $^{17}$, radiculopatia ${ }^{4,5,6,14}$, lesão óssea e de partes moles ${ }^{4,5}$.

Schoenen et al. ${ }^{14}$ descreveram quatro pacientes com provável envolvimento central por apresentar discinesias faciais associadas ao quadro; em três desses pacientes, o componente doloroso era mais brando, e ainda um deles apresentava TC com alargamento do sistema ventricular e uma cisternocintilografia que sugeria hidrocefalia de pressão compensada. Leger $e t$ $a l .{ }^{11}$ descreveram o caso de um paciente com PLMT associado a espasmo hemifacial, sincinesias oculolabiais e reflexo cutâneo-plantar em extensão, também mostrando evidência de lesão central. 
O mecanismo fisiopatológico ainda é controverso. Shaibani et $a l .^{2}$ descreveram um paciente com movimentos involuntários como os descritos por Spillane et al. ${ }^{1}$, porém sem dor e associados à neuropatia hereditária sensível a pressão e a outros movimentos anormais, tais como distonia e mioclonia. Os autores sugeriram que os movimentos anormais seriam devidos a um mecanismo central induzido pela patologia periférica. Tan e $\operatorname{Tan}^{16}$ concordam com essa hipótese baseados em Dressler et $a l .^{4}$, os quais citam as mudanças que uma lesão no nervo periférico poderia causar uma ativação neuronal no corno posterior, nos núcleos da coluna dorsal ventral do tálamo e no córtex somatossensorial. Schott ${ }^{5}$ apóia a idéia de que microtraumas periféricos poderiam disseminar um estímulo irritativo para a medula espinhal, o que envolve fibras sensitivas, motoras e autonômicas, traduzindo-se na síndrome de PLMT.

Nathan ${ }^{8}$ propôs que os movimentos coordenados dos artelhos são causados pela ativação de interneurônios medulares por estímulos vindos das raízes posteriores. Já Pla et al. ${ }^{15}$ propõem, no caso de seu paciente que possuía uma síndrome do túnel do tarso associada com PLMT, que o mecanismo gerador dos movimentos e da dor seja periférico, pois bloqueios abaixo do nível da compressão podiam suprimir os movimentos, enquanto os acima não tinham o mesmo efeito. Mitsumoto et al. ${ }^{18}$ descreveram um paciente com mononeurite hipertrófica e propuseram que essa lesão periférica é eletricamente irritável, causando os movimentos anormais. Walters et al. ${ }^{3}$ descrevem a síndrome painless leg and moving toes sem evidência de lesão central ou periférica. Esses autores também discutem a possibilidade de tratar-se de síndrome de etiologia diversa da PMLT ou de uma forma frustra. Schoenen et al. ${ }^{14}$ acreditam que PMLT seja uma síndrome com mecanismos fisiopatológicos diferentes, podendo ser central ou periférica. Para ajudar a diferenciar os dois grupos, mesmo sabendo que não se trata de sinais específicos, propõe-se a observação da intensidade da dor, da presença de cãibras, da evidência de radiculopatia ou neuropatia e de discinesias. Sugerem que a ENMG seja essencial para distinguir se uma lesão é periférica ou central. Quando periférica, a ENMG mostra descargas espontâneas com amplitude pequena $(100 \mu \mathrm{V}$ a $2 \mu \mathrm{V})$, freqüência irregular $(4 \mathrm{~Hz}$ a $6 \mathrm{~Hz})$ e curta duração $(10 \mathrm{~m} / \mathrm{s}$ a $80 \mathrm{~m} / \mathrm{s}$ ), vistas sincronicamente em músculos antagonistas; quando central, mostra descargas de grande amplitude $(1 \mu \mathrm{V}$ a $3 \mu \mathrm{V})$, com freqüência mais lenta $(1,5 \mathrm{~Hz}$ a $3 \mathrm{~Hz})$ e longa duração $(160 \mathrm{~m} / \mathrm{s}$ a $500 \mathrm{~m} / \mathrm{s}$ ), alternando em músculos antagonistas. Em nosso paciente, observamos descargas espontâneas, esporádicas, de freqüência irregular, baixa amplitude, vistas sincronicamente em músculos antagonistas, sugerindo um acometimento periférico segundo esse critério.

Várias tentativas terapêuticas já foram feitas. Algum sucesso foi observado com o uso de baclofeno $^{4,11,14,16,19}$ e com clonazepam ${ }^{16,19,20}$. Shaibani et $a l .^{2}$ não obtiveram boa resposta com clonazepam, mas o paciente apresentou discreta melhora com triexifenidila. Analgésicos, esteróides, vitamina B12, propanolol, ACTH, drogas parassimpatolíticas, quinino, frio local, injeções de anestéticos locais, carbamazepina, anticolinérgicos, anti-serotoninérgicos, L-dopa, amantadina, benzodiazepínicos, neurolépticos, tetrabenazina, milacemida, antidepressivos, vibração, estimulação elétrica transcutânea, acupuntura e bloqueio simpático foram tentados, sem, contudo, obter uma resposta satisfatória ${ }^{1,4,5,7,8,11,13,14,20,21}$. Gastaut ${ }^{9}$, Malapert e Degos ${ }^{10}$, assim como nós, obtiveram boa resposta com a carbamazepina. Bovier et al. ${ }^{22}$ utilizaram progabide em um paciente, obtendo resultados satisfatórios. Guieu et al..$^{7}$ utilizaram estimulação combinada vibratória e elétrica transcutânea com alívio da dor e desaparecimento dos movimentos. Leger et al. ${ }^{11}$ obtiveram um bom efeito com o uso de reserpina em um paciente com provável origem central para a síndrome.

Nosso paciente apresentou os sintomas da síndrome descrita por Spillane ${ }^{1}$. Mesmo se tratando de uma paciente diabética, não observamos anormalidades na sua ENMG típicas de uma neuropatia diabética, porém as descargas espontâneas encontradas falam a favor de origem periférica para a sintomatologia apresentada segundo critério de Schoenen et al. ${ }^{14}$. Os achados de imagem obtidos nesse caso, por outro lado, apontam para um acometimento do sistema nervoso central. O envolvimento central com isquemia e atrofia de núcleos da base não costuma estar associado a PLMT e esse achado de nosso paciente poderia ser uma coincidência, já que também isquemias lacunares podem ser assintomáticas em muitos indivíduos. De qualquer modo, também podemos supor que a combinação dos achados (central e periférico) poderia favorecer o desenvolvimento do quadro clínico. O envolvimento central e periférico em um mesmo paciente, como descrito em nosso paciente, também pode ser uma das causas de PLMT. 


\section{SUMMARY}

Painful legs and moving toes syndrome associated with alterations in the basal ganglia nuclei

We report on a female patient with the syndrome of painful legs and moving toes. The patient was submitted to electroneuromyography, tomography and magnetic resonance image of the head. The electroneuromyography (EMG) showed no abnormalities, however the EMG pattern of movements suggested a periferal disorder. The image examinations showed atrophy in the left side basal ganglia nuclei and hypodense lesions in the white matter suggestive of lacunary infarcts. If it is consider that this syndrom could be caused by central or peripheric disorders, we suggest that this patient had both etiologies. The response to carbamazepine was successful in relieving her pain as well as almost eliminating the involuntary movements.

\section{Keyw ords}

Painful legs, involuntary movements, basal ganglia nuclei.

\section{Referências}

1. Spillane JD, Nathan PW, Kelly RE, Marsden CD. Painful legs and moving toes. Brain, 94(3):541-56, 1971.

2. Shaibani A, Gooch C, Harati Y. Moving toes and myoclonus associated with hereditary neuropathy with liability to pressure palsy (HNPP). Muscle Nerve, 20(7):881-3, 1977.

3. Walters AS, Hening WA, Shah SK, Chokroverty S. Painless legs and moving toes: a syndrome related to painful legs and moving toes? Mov Disord, 8(3):377-9, 1993.

4. Dressler D, Thompson PD, Gledhill RF, Marsden CD. The syndrome of painful legs and moving toes. Mov Disord, 9(1):13-21, 1994.

5. Schott GD. Painful legs and moving toes: the role of trauma. J Neurol Neurosurg Psychiatry, 44(4):344-6, 1981.

6. Wulff $\mathrm{CH}$. Painful legs and moving toes. A report of 3 cases with neurophysiological studies. Acta Neurol Scand, 66(2):283-7, 1982.

7. Guieu R, Tardy-Gervet MF, Blin O, Pouget J, Guy B. Pain relief achived by transcutaneous electrical nerve stimulation and/or vibratory stimulation in a case of painful legs and moving toes. Pain, 42(1):43-8, 1990.
8. Nathan PW. Painful legs and moving toes: evidence on the site of the lesion. J Neurol Neurosurg Psychiatry, 41(10):934-9, 1978.

9. Gastaut JL. Painful legs and moving toes. A drug-induced case. Rev Neurol (Paris), 142(6-7):641-2, 1986.

10. Malapert D, Degos JD. Painful legs and moving toes. Neuropathy caused by cytarabine. Rev Neurol (Paris), 145(12):869-71, 1989.

11. Leger JM, Lubetzki C, Bouche P, Bor Y, Brunet P. Painful legs and unstable toes. Rev Neurol (Paris), 141(4):296304, 1985.

12. Sahashi K, Tsuchiya I, Iwase S, Ibi T, Mano T. Clinical analyses on moving toes in painful legs and moving toes. Rinsho Shinkeigaku, 29(7):849-53, 1989.

13. Montagna $P$, Cirignotta $F$, Sacquegna $T$, Martinelli $P$, Ambrosetto G, Lugaresi E. Painful legs and moving toes associated with polyneuropathy. J Neurol Neurosurg Psychiatry, 46(5):399-403, 1983.

14. Schoenen J, Gonce M, Delwaide PJ. Painful legs and moving toes: a syndrome with different physiopathologic mechanisms. Neurology, 34(8):1108-12, 1984.

15. Pla ME, Dillingham TR, Spellman NT, Colon E, Jabbari B. Painful legs and moving toes associates with tarsal tunnel syndrome and accessory soleus muscle. Mov Disord, 11(1):82-6, 1996.

16. Tan AK \& Tan CB. The syndrome of painful legs and moving toes - a case report. Singapore Med J, 37(4):4467, 1996.

17. Scherokman B, Jabbari B, Ling G. Painful legs and moving toes - favorable response to lioresal and association with HIV infection [abstract]. Neurology, 41:245, 1991.

18. Mitsumoto $\mathrm{H}$, Levin $\mathrm{KH}$, Wilbourn AJ, Chou SM. Hypertrophic mononeuritis clinically presenting with painful legs and moving toes. Muscle Nerve, 13(3):21521, 1990.

19. Sandyk R. Neuroleptic-induced painful legs and moving toes syndrome: successful treatment with clonazepam and baclofen. Ital J Neurol Sci, 11(6):573-6, 1990.

20. Sichere P, Valade D, Morizot-Koutlidis R, Margot-Duclot A. Painful legs and moving toes syndrome. Apropos of 2 new cases. Rev Rhum Mal Osteoartic, 57(1):25-8, 1990.

21. Lance JW, Andrews C. Dysaesthesia-dyskinesia: a syndrome of painful legs and moving toes. Proc Aust Assoc Neurol, 9:87-90, 1973.

22. Bovier $\mathrm{P}$, Hilleret $\mathrm{H}$, Tissot $\mathrm{R}$. Progabide treatment of a case of the syndrome of painful legs and moving toes. Rev Neurol (Paris), 141(5):422-4, 1985.

Endereço para correspondência:

Dra. Thaís Minett

Av. Conselheiro Rodrigues Alves, 890, ap. 61

CEP 04014-010 - São Paulo, SP 